\title{
Rechallenge with pemetrexed-based chemotherapy improves the survival of patients with advanced non-squamous non-small-cell lung cancer
}

\author{
MING-LEI ZHUO, HUA BAI, ZHI-JIE WANG, JIAN-CHUN DUAN, TONG-TONG AN, MEI-NA WU, \\ JUN ZHAO, YU-YAN WANG, SHU-HANG WANG and JIE WANG \\ Department of Thoracic Medical Oncology, Peking University School of Oncology, \\ Beijing Cancer Hospital and Institute, Beijing 100142, P.R. China
}

Received February 27, 2014; Accepted March 26, 2014

DOI: $10.3892 / \mathrm{mco} .2014 .359$

\begin{abstract}
Rechallenge chemotherapy with pemetrexed was shown to be efficient in malignant pleural mesothelioma; however, its role in non-small-cell lung cancer (NSCLC) has not been investigated. In this study, we retrospectively enrolled 31 patients with non-squamous NSCLC who had achieved disease control with initial pemetrexed treatment, followed by rechallenge with pemetrexed-based chemotherapy (PBC) upon disease progression. After the rechallenge, 5 patients (16.1\%) achieved partial remission (PR), 17 (54.8\%) achieved stable disease (SD) and $9(29.1 \%)$ experienced progressive disease. The treatment was generally well tolerated, with a low rate of toxicity. The median progression-free survival (PFS) was 3.8 months with the rechallenge. Patients with a PFS of $\geq 10$ months with initial PBC exhibited longer PFS and overall survival (OS) with the rechallenge compared to those with a PFS of $<10$ months with initial PBC (PFS: $6.2 \pm 0.33$ vs. $3.1 \pm 0.26$ months, respectively; $\mathrm{P}=0.011$; and $\mathrm{OS}$, $19.8 \pm 3.2$ vs. $9.2 \pm 1.1$ months, respectively; $\mathrm{P}=0.005)$. The time from the termination of initial $\mathrm{PBC}$ to disease progression was also associated with survival after the rechallenge. However, the response to initial PBC (PR vs. SD) did not affect PFS after the rechallenge. No significant differences were observed in thymidylate synthase expression, echinoderm microtubule-associated protein-like 4-anaplastic lymphoma kinase gene fusion, or epithelial growth factor receptor mutation status between pemetrexed-sensitive and pemetrexed-resistant patients. Our results demonstrated that rechallenge with PBC was well tolerated and survival after the rechallenge was associated with survival during initial PBC. Therefore, patients with
\end{abstract}

Correspondence to: Dr Jie Wang, Department of Thoracic Medical Oncology, Peking University School of Oncology, Beijing Cancer Hospital and Institute, 52 Fucheng Road, Haidian District, Beijing 100142, P.R. China

E-mail: zlhuxi@163.com

Key words: pemetrexed, rechallenge, non-small-cell lung cancer, overall survival, adverse effects a PFS of $\geq 10$ months or time-to-disease progression $\geq 3$ months may be considered as candidates for pemetrexed rechallenge.

\section{Introduction}

Pemetrexed is a potent chemotherapeutic agent with known high efficacy and relatively low toxicity in the treatment of non-squamous non-small-cell lung cancer (NSCLC) and malignant pleural mesothelioma (MPM). As first-line therapy for advanced lung adenocarcinoma, pemetrexed-based chemotherapy (PBC) has yielded an average overall survival (OS) of 12.6 months (1). As second-line therapy for advanced NSCLC, pemetrexed has yielded an overall response rate of $9.1 \%$, a median progression-free survival (PFS) of 2.9 months and a median survival time of 8.3 months (2). As first-line therapy for MPM, the median survival time with pemetrexed/cisplatin treatment was 12.1 months and the response rate was significantly higher in the pemetrexed/cisplatin group compared to that in the control group (41.3 vs. $16.7 \%$, respectively; $\mathrm{P}<0.0001$ ) (3).

Maintenance therapy with pemetrexed is an effective strategy and is recommended as standard treatment for patients who achieve disease control following first-line chemotherapy with 4-6 cycles of platinum-based doublet chemotherapy regimens. However, a considerable number of patients are not administered pemetrexed maintenance therapy due to concerns regarding toxicity or high treatment cost. Therefore, it is imperative to investigate $\mathrm{PBC}$ as potential second-line therapy upon disease progression following first-line chemotherapy.

It was recently demonstrated that $\mathrm{PBC}$ may be used as rechallenge treatment in MPM patients who achieve a $\mathrm{PFS} \geq 12$ months with first-line PBC (4-6). We have also noticed in clinical practice that certain patients with non-squamous NSCLC benefited from rechallenge with PBC as second- or further-line treatment. Therefore, the aim of this study was to investigate the efficacy and safety of rechallenge with $\mathrm{PBC}$ in patients with advanced non-squamous NSCLC.

\section{Materials and methods}

Patient selection. We evaluated patients with histologically confirmed advanced non-squamous NSCLC who underwent 
PBC rechallenge as second- or further-line therapy. The presence of unidimensionally measurable disease was mandatory for inclusion in the study. The eligibility criteria also included age $\geq 18$ years, Eastern Cooperative Oncology Group (ECOG) performance status (PS) $\leq 2$ and an estimated life expectancy of $\geq 3$ months. An adequate bone marrow reserve was required, with absolute neutrophil count $\geq 2.0 \times 10^{9} / 1$, platelet count $\geq 100 \times 10^{9} / 1$ and hemoglobin $\geq 9 \mathrm{~g} / \mathrm{dl}$. Creatinine clearance $\geq 60 \mathrm{ml} / \mathrm{min}$, bilirubin $\leq 1.5$-fold the upper limit of normal (ULN) and alanine aminotransferase or asparate aminotransferase (AST) $\leq 3$-fold ULN were also required, whereas AST was allowed to be $\leq 5$-fold ULN in patients with known hepatic metastases. Patients with severe comorbidities or other malignancies were excluded from this study. Written informed consent was obtained from each patient prior to the initiation of the PBC rechallenge. This study conformed to the ethical rules of the Declaration of Helsinki and the protocol was approved by the Institutional Review Board of Beijing Cancer Hospital and Institute.

Study design. We conducted a retrospective study on PBC rechallenge in a consecutive series of patients who had received initial PBC in the Department of Thoracic Medical Oncology at the Beijing Cancer Hospital. This was an observational study, thus, no statistical design was used. We conducted descriptive analyses of PFS, OS, tumor response rate and toxicity.

Treatment. The PBC regimens included single-agent pemetrexed $\left(500 \mathrm{mg} / \mathrm{m}^{2}\right.$ every 3 weeks) and pemetrexed plus a platinum compound, such as carboplatin (area under the plasma concentration time curve of $5 \mathrm{mg} / \mathrm{ml} / \mathrm{min}$ administered on day 1 of every 21 -day cycle) or cisplatin $\left(75 \mathrm{mg} / \mathrm{m}^{2}\right.$ administered on days 1-2 of every 21-day cycle). All the patients received supplemental folic acid and vitamin B12. Standard prophylaxis for nausea and vomiting was also administered as dexamethasone and intravenous 5-hydroxytryptamine type 3 receptor antagonists. The chemotherapy dose was reduced by $20 \%$ for patients who experienced grade 4 toxicity. Platinum-containing regimens were repeated for a maximum of 6 cycles; eligible patients were then allowed to switch to single-agent pemetrexed maintenance therapy, which was continued until disease progression or unacceptable toxicity.

Patient assessment. The baseline patient assessment included a complete medical history, physical examination, complete blood count (CBC) and blood chemistry tests. CBC and blood chemistry tests were obtained prior to each course of chemotherapy. A chest computed tomography (CT) scan, cerebral magnetic resonance imaging and cervical and abdominal ultrasound (US) examinations were performed at baseline and after every 2 cycles of treatment. An abdominal CT scan was required if the patient had developed abdominal metastases. After completion of the study treatment, the patients were evaluated every 3 months with chest CT and cervical and abdominal US scans until disease progression. The patients were observed for survival until death or last contact. The optimal tumor response was evaluated according to the Response Evaluation Criteria in Solid Tumors, version 1.1 (7). Treatment toxicity was evaluated according to the National Cancer Institute Common Toxicity Criteria, version 4.0 (http://evs.nci.nih.gov/ftp1/CTCAE/ CTCAE_4.03_2010-06-14_QuickReference_5x7.pdf).
Sample collection and processing. DNA was extracted from formalin-fixed paraffin-embedded (FFPE) tumor tissues using E.Z.N.A. FFPE DNA kits (Omega Bio-Tek, Inc., Norcross, GA, USA). The quality and concentration of the extracted DNA were determined using NanoDrop 2000 (Thermo Fisher Scientific, Wilmington, DE, USA). The extracted DNA was then used for epithelial growth factor receptor (EGFR) mutation analysis by denaturing high-performance liquid chromatography (8). The thymidylate synthase (TS) protein expression status was assessed by immunohistochemistry using Thymidylate Synthase (D5B3) XP ${ }^{\circledR}$ Rabbit mAb (Cell Signaling Technology, Danvers, MA, USA). Immunohistochemical staining was performed according to the manufacturer's instructions. Two pathologists independently quantified the staining intensity, which was graded on a scale from $0^{+}$to $3^{+}$; the percentage of tumor cells was noted within each intensity category. The percentage score was then multiplied by its intensity category to obtain a final H-score, which ranged from 0 to 300 . The highest $\mathrm{H}$-score of triplicate scores for each patient was used for further analyses.

Echinoderm microtubule-associated protein-like 4-anaplastic lymphoma kinase (EML4-ALK) fusion detection. Fluorescence in situ hybridization (FISH) was performed on FFPE tumor tissues using the Vysis LSI ALK Dual Color Break Apart Rearrangement Probe (Abbott Molecular, Abbott Park, IL, USA). The assays were performed according to the manufacturer's instructions. The tumor sections were analyzed under a fluorescence microscope equipped with a triple-pass filter (DAPI/Green/Orange). A FISH-positive sample was defined as $15 \%$ of tumor cells with split signals.

Statistical analysis. PFS was calculated as the time from the first day of study treatment until disease progression, as indicated by radiological or clinical examination, or death from any cause. Patients without any evidence of progressive disease (PD) were censored at the date of the last follow-up. OS was defined as the time from the first day of study treatment until death from any cause; patients who remained alive on the date of the last follow-up were censored on that date. If survival status was unknown at the final follow-up, the OS time was censored at the last contact date. OS and PFS analyses were computed by the Kaplan-Meier method. In all the cases, statistical significance was established as $\mathrm{P} \leq 0.05$. Statistical analyses were performed with the SPSS 13.0 software package (SPSS Inc., Chicago, IL, USA).

\section{Results}

Clinical characteristics and treatment regimens. Between January, 2009 and June, 2013, a total of 31 patients underwent a $\mathrm{PBC}$ rechallenge in our department. Of the 31 patients, 16 were male and 15 were female. The median age was 58.5 years (range, $37-83$ years). Of the 31 patients, 30 were diagnosed with adenocarcinoma and 1 patient was diagnosed with undifferentiated non-squamous NSCLC. A total of 19 patients received initial $\mathrm{PBC}$ as first-line therapy and 12 received $\mathrm{PBC}$ as second- or further-line therapy. A total of 11 patients received the $\mathrm{PBC}$ rechallenge as second-line therapy and 20 patients received the rechallenge as third- or 
further-line therapy. The clinical characteristics of the patients are summarized in Table I.

The initial $\mathrm{PBC}$ regimens included pemetrexed plus carboplatin (8 cases), pemetrexed plus cisplatin (17 cases) and pemetrexed as single-agent therapy (6 patients). A total of 19 patients achieved a partial response (PR) and 12 patients achieved stable disease (SD) following initial PBC. The median PFS following initial PBC (PFS1) was 10.2 months (range, 1-23 months).

The $\mathrm{PBC}$ rechallenge regimens included pemetrexed as single-agent therapy (11 cases), pemetrexed plus carboplatin ( 8 cases) and pemetrexed plus cisplatin (12 cases). A median of 4 cycles was administered (range, 2-8 cycles). Intermediate regimens, which were administered between initial $\mathrm{PBC}$ and PBC rechallenge, included single-agent docetaxel, gemcitabine and EGFR-tyrosine kinase inhibitors (TKIs). A total of 7 patients received further-line therapies following disease progression after $\mathrm{PBC}$ rechallenge, including 2 patients who received an additional line of $\mathrm{PBC}$.

Outcomes of $\mathrm{PBC}$ rechallenge. After the $\mathrm{PBC}$ rechallenge, 5 patients $(16.1 \%)$ achieved a PR, 17 patients $(54.8 \%)$ achieved SD and 9 patients (29.1\%) experienced PD. Overall, the disease control rate was $70.9 \%$. Rechallenge with PBC was generally well tolerated. Grade 3/4 hematological toxicity was observed in 6 patients $(19.4 \%$ ), grade $1 / 2$ fatigue was observed in 8 patients $(25.8 \%)$, grade 3 gastrointestinal upset was reported in 1 patient and grade 1 proteinuria was observed in 1 patient. However, unlike single-agent pemetrexed treatment, the doublet regimens were associated with more severe fatigue, bone marrow depression and gastrointestinal adverse effects, which led to treatment discontinuation in 4 patients.

The patients were followed up for a median of 34 months. During follow-up, 18 patients succumbed to the disease. Of the 13 patients who remained alive on the date of the last follow-up, 4 had no evidence of disease progression. The median PFS for all the patients who received a PBC rechallenge was 3.8 months (range, 1.3-15.1 months). There were no significant differences in the overall response rate or PFS between single-agent and combined chemotherapy regimens for the rechallenge $(\mathrm{P}=0.129$ and $\mathrm{P}=0.201$, respectively).

The PFS achieved after the PBC rechallenge (PFS2) was correlated with PFS1. Patients with a PFS1 of $\geq 10$ months had a median PFS2 of $6.2 \pm 0.33$ months, whereas patients with a PFS1 of $<10$ months had a median PFS2 of $3.1 \pm 0.26$ months $(\mathrm{P}=0.011)$ (Fig. 1A). The OS for the rechallenge was also correlated with PFS1. Patients with a PFS1 of $\geq 10$ months had a longer OS compared to those with a PFS1 of $<10$ months (median OS: $16.47 \pm 3.2$ vs. $8.83 \pm 1.24$ months, respectively; $\mathrm{P}=0.005$ ) (Fig. 1B).

The treatment-free survival (TFS), calculated from the last dose of the initial $\mathrm{PBC}$ to radiologic evidence of disease progression, was also associated with survival after the rechallenge. Patients with a TFS of $\geq 3$ months had a longer PFS2 compared to those with a TFS of $<3$ months (median PFS2: $5.5 \pm 0.90$ vs. $2.10 \pm 0.28$ months, respectively; $\mathrm{P}=0.001$ ) (Fig. 1C). Patients with a TFS of $\geq 3$ months tended to have a longer OS compared to patients with a TFS of $<3$ months, although statistical significance was not reached (median OS: $13.10 \pm 1.93$ vs. $10.70 \pm 2.79$ months, respectively; $\mathrm{P}=0.198)$ (Fig. 1D).
Table I. Patient characteristics.

\begin{tabular}{|c|c|c|}
\hline Characteristics & $\begin{array}{l}\text { No. of patients } \\
\qquad(\mathrm{n}=31)\end{array}$ & $\%$ \\
\hline \multicolumn{3}{|l|}{ Age, years } \\
\hline Median (range) & $58.5(37-83)$ & \\
\hline \multicolumn{3}{|l|}{ Gender } \\
\hline Male & 16 & 51.6 \\
\hline Female & 15 & 48.4 \\
\hline \multicolumn{3}{|l|}{ Histotype } \\
\hline Adenocarcinoma & 30 & 96.8 \\
\hline Undifferentiated & 1 & 3.2 \\
\hline \multicolumn{3}{|c|}{ ECOG performance status } \\
\hline 0 & 0 & 0.0 \\
\hline 1 & 30 & 96.8 \\
\hline 2 & 1 & 3.2 \\
\hline \multicolumn{3}{|c|}{ Response to initial PBC } \\
\hline PR & 19 & 61.3 \\
\hline SD & 12 & 38.7 \\
\hline \multicolumn{3}{|c|}{ Response to $\mathrm{PBC}$ rechallenge } \\
\hline PR & 5 & 16.1 \\
\hline $\mathrm{SD}$ & 17 & 54.8 \\
\hline PD & 9 & 29.1 \\
\hline \multicolumn{3}{|c|}{ PFS after initial PBC, months } \\
\hline$<10$ & 14 & 45.2 \\
\hline$\geq 10$ & 17 & 54.8 \\
\hline \multicolumn{3}{|c|}{ TFS after initial PBC, months } \\
\hline$<3$ & 8 & 25.8 \\
\hline$\geq 3$ & 23 & 74.2 \\
\hline \multicolumn{3}{|c|}{ Line of therapy with rechallenge } \\
\hline 2nd & 11 & 35.5 \\
\hline $3 r d$ & 7 & 22.6 \\
\hline$\geq 4$ th & 13 & 41.9 \\
\hline
\end{tabular}

ECOG, Eastern Cooperative Oncology Group; PBC, pemetrexed-based chemotherapy; PFS, progression-free survival; PR, partial response; $\mathrm{SD}$, stable disease; $\mathrm{PD}$, progressive disease; TFS, treatment-free survival.

The response to initial PBC (PR vs. SD) did not affect PFS2 or OS after the PBC rechallenge (median PFS2: $5.00 \pm 1.03$ vs. $3.20 \pm 0.39$ months, respectively; $\mathrm{P}=0.598$; and median OS: $12.30 \pm 0.96$ vs. $10.70 \pm 4.01$ months, respectively; $\mathrm{P}=0.589$ ) (Fig. 2).

Molecular analysis and PBC rechallenge. Molecular markers were analyzed in the 31 patients in this study who were classified as sensitive to PBC. The EGFR mutation rate (exons 19,21) was $25.8 \%(n=8)$. Of the 10 patients who provided available FFPE sections for further analysis, 2 cases were positive for EML4-ALK gene fusion and 4 patients exhibited positive staining for TS, which were considered potential predictors of pemetrexed efficacy $(9-11)$. 

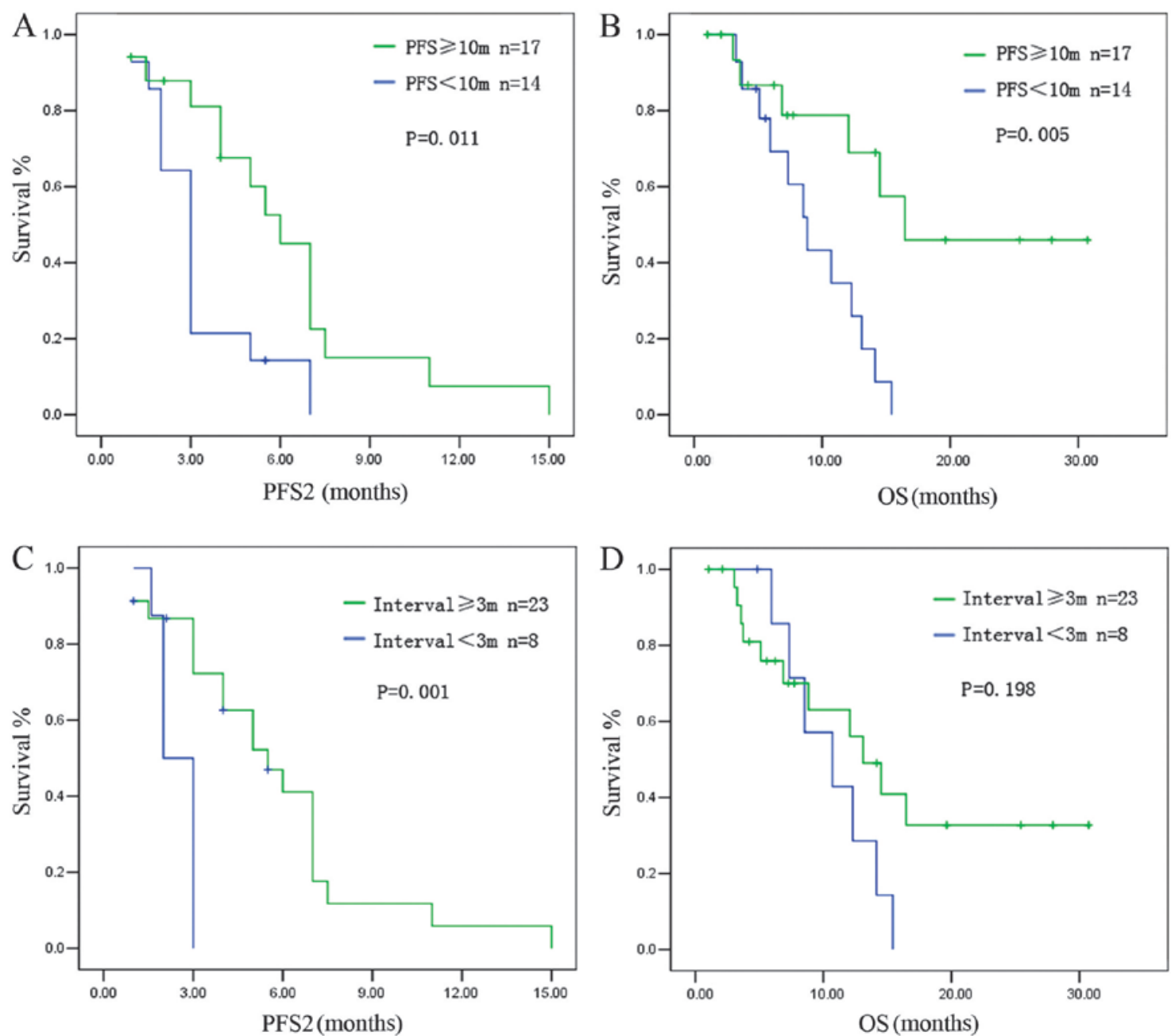

Figure 1. Survival of patients who received pemetrexed-based chemotherapy (PBC) rechallenge. (A) Patients with a progression-free survival after initial PBC (PFS1) of $\geq 10$ months had a longer PFS after the PBC rechallenge (PFS2) compared to those with a PFS1 of $<10$ months. (B) Patients with a PFS1 of $\geq 10$ months had a longer overall survival (OS) compared to those with a PFS1 of $<10$ months. (C) Patients with a treatment-free survival (TFS) of $\geq 3$ months had a longer PFS2 compared to those with a TFS of $<3$ months. (D) Patients with a TFS of $\geq 3$ months tended to have a longer OS compared to those with a TFS of $<3$ months.
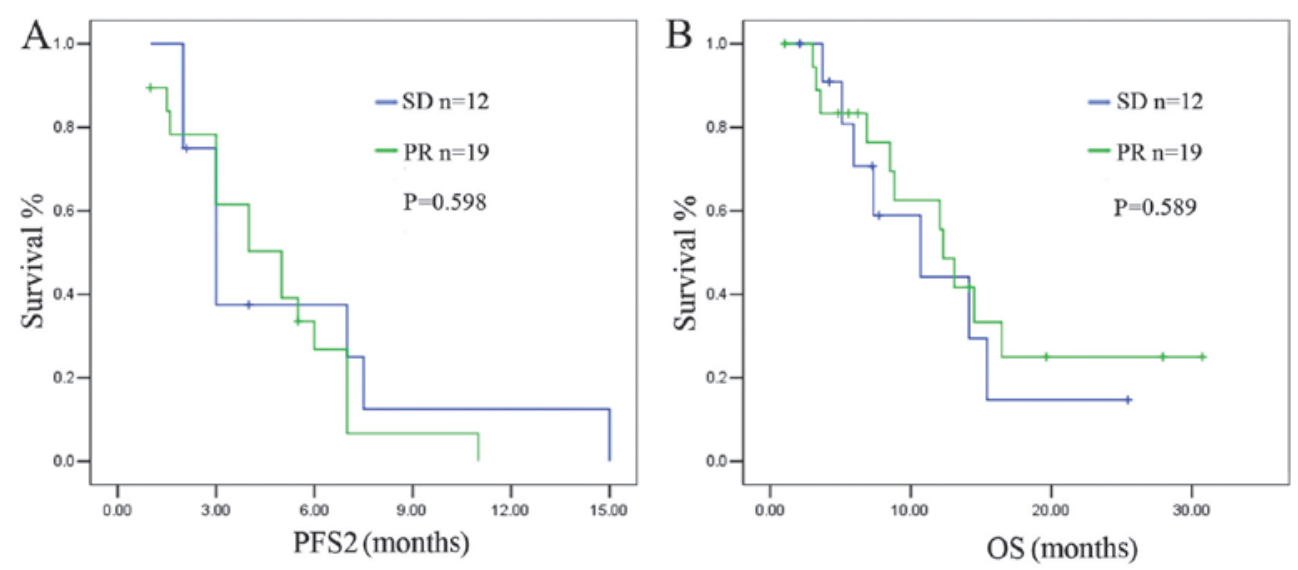

Figure 2. Association of response to initial pemetrexed-based chemotherapy (PBC) and survival after the PBC rechallenge. No significant differences were observed in (A) the progression-free survival after the PBC rechallenge (PFS2) or (B) the overall survival (OS) after the PBC rechallenge between the partial response (PR) and stable disease (SD) subgroups after initial treatment with PBC.

We analyzed the same markers in another group of patients $(\mathrm{n}=55)$ who were primarily resistant to $\mathrm{PBC}$ and experienced PD within 4 cycles of initial treatment with PBC. The EGFR mutation rate in this group was $29.1 \%(n=16)$. Of the 21 cases submitted for further analysis, 7 patients exhibited positive TS expression and 2 patients were positive for EML4-ALK gene fusion (Table II). No significant differences were observed between the PBC-sensitive and PBC-resistant groups. 

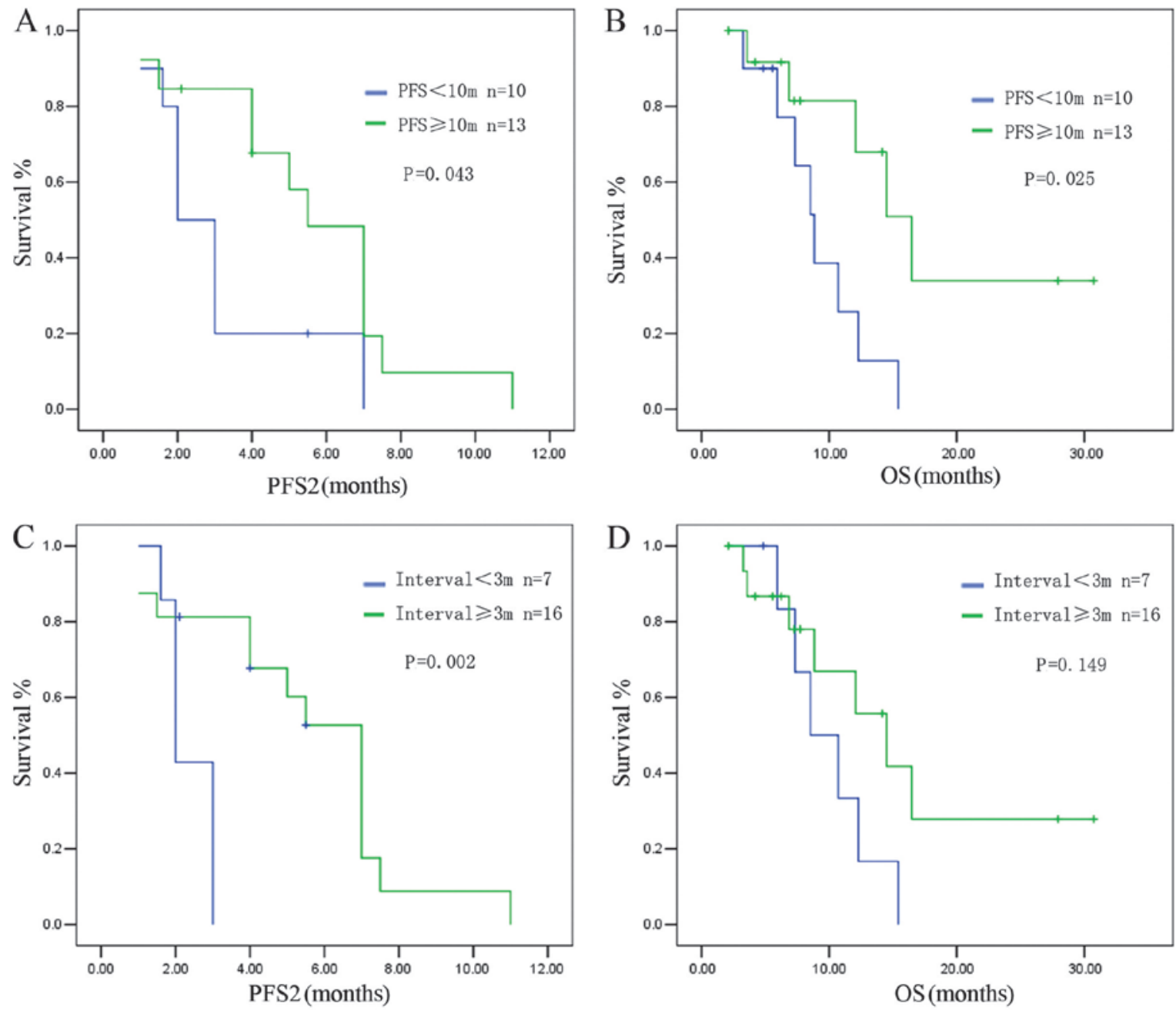

Figure 3. Survival of epithelial growth factor receptor (EGFR) wild-type patients. (A) Patients with a progression-free survival after initial pemetrexed-based chemotherapy (PFS1) of $\geq 10$ months exhibited a longer PFS after the rechallenge (PFS2) compared to those with a PFS1 of $<10$ months. (B) Patients with a PFS1 of $\geq 10$ months had a longer overall survival (OS) compared to those with a PFS1 of $<10$ months. (C) Patients with a treatment-free survival (TFS) of $\geq 3$ months exhibited a longer PFS 2 compared to those with a TFS of $<3$ months. (D) Patients with a TFS of $\geq 3$ months tended to have a longer OS compared to those with a TFS of $<3$ months.

Table II. Molecular analyses.

\begin{tabular}{|c|c|c|}
\hline Markers & Group $\mathrm{A}^{\mathrm{a}}$ & Group B ${ }^{b}$ \\
\hline EGFR & $25.8 \% \quad(8 / 31)$ & $29.1 \%(16 / 55)$ \\
\hline TS & $40 \% \quad(4 / 10)$ & $30 \% \quad(7 / 21)$ \\
\hline EML4-ALK & $20 \% \quad(2 / 10)$ & $9.5 \% \quad(2 / 21)$ \\
\hline
\end{tabular}

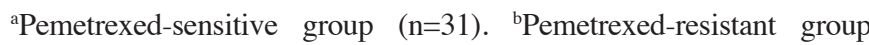
$(\mathrm{n}=55)$; EGFR, epithelial growth factor receptor; TS, thymidylate synthase; EML4-ALK, echinoderm microtubule-associated protein-like 4-anaplastic lymphoma kinase. Data are presented as positive rate observed in the analysis (positive case number/total cases analyzed).

The EGFR mutation status may affect the clinical outcome of PBC; therefore, we subsequently analyzed the subgroup of 23 EGFR wild-type patients in the original study group of 31 patients (we excluded the 8 patients with EGFR-sensitive mutations). The results were similar to those observed in the entire group.
Patients with a PFS1 of $\geq 10$ months had a median PFS2 of $5.52 \pm 0.77$ months, whereas patients with a PFS1 of $<10$ months had a median PFS2 of $2.13 \pm 0.36$ months $(\mathrm{P}=0.043)$ (Fig. $3 \mathrm{~A})$. A longer OS was observed in patients with a PFS1 $\geq 10$ months compared to patients with a PFS1 of $<10$ months (median OS: $16.47 \pm 2.42$ vs. $8.83 \pm 1.01$ months, respectively; $\mathrm{P}=0.025$ ) (Fig. 3B).

Patients with a TFS of $\geq 3$ months exhibited a longer PFS2 after the PBC rechallenge compared to those with a TFS of $<3$ months (PFS2: $7.2 \pm 0.52$ vs. $2.0 \pm 0.18$ months, respectively; $\mathrm{P}=0.002$ ) (Fig. 3C). The OS also tended to be longer in the group with a longer TFS interval, although statistical significance was not reached (OS: $14.5 \pm 2.94$ vs. 8.53 \pm 2.06 months, respectively; $\mathrm{P}=0.149$ ) (Fig. $3 \mathrm{D}$ ).

\section{Discussion}

Malignancies such as ovarian cancer and SCLC, may be retreated with the first-line chemotherapeutic regimen if a patient develops a sensitive relapse, which is defined as disease that responds to first-line chemotherapy, but relapses 
$>6$ months after the last dose of the first-line treatment $(12,13)$. However, in NSCLC, there are currently no available studies on rechallenging with certain first-line regimens.

In this study, we analyzed a total of 31 NSCLC patients who underwent rechallenge with PBC. All the patients had achieved disease control after the initial PBC. Rechallenging with PBC was associated with acceptable tolerability, while doublet regimens are generally associated with more severe adverse effects, including bone marrow depression, liver damage and fatigue. Our results suggested that combined PBC regimens should be selectively reserved for patients with good PS scores and who have experienced few toxicities with prior chemotherapy regimens.

We observed that the patients who achieved a PFS1 of $\geq 10$ months exhibited a longer PFS2 and OS compared to those with a PFS1 of $<10$ months. Patients with a TFS of $\geq 3$ months also exhibited a longer PFS2 compared to those with a TFS of $<3$ months. Such results are similar to those reported by previous studies on MPM patients $(4,6)$. However, the cut-off value of PFS1 in this study was shorter compared to that in the study of Ceresoli et al (4) (10 vs. 12 months, respectively). This difference may be attributed to the differences in the physical condition of the patients. In the Ceresoli study, all the patients received PBC as first-line therapy; the PS score was 0 in 12 patients $(38.7 \%)$ and 1 in 18 patients $(58.1 \%)$. However, in the present study, 12 patients (38.7\%) received PBC as second- or further-line therapy and the PS score was 1 in 30 patients and 2 in 1 patient. This gap in the physical condition of the patients is likely to have caused the difference in the cut-off value of PFS1 between the two studies.

EGFR-TKIs play an important role in the treatment of advanced non-squamous NSCLC and TKI therapy may significantly affect OS. We observed that, in the subgroup with a PFS1 of $<10$ months, 4 patients were EGFR mutation-positive, 13 patients received EGFR-TKI therapy and the median PFS was 5.38 months; in the subgroup with PFS $1 \geq 10$ months, 4 patients were EGFR mutation-positive, 9 patients received EGFR-TKI therapy and the median PFS was 4.33 months. No significant differences were observed between these two subgroups. Furthermore, the PBC rechallenge also increased survival among EGFR wild-type patients, indicating that the survival results were likely not confounded by EGFR-TKI therapy.

Continuous maintenance treatment with pemetrexed is an effective and well-tolerated option for patients with advanced non-squamous NSCLC with good PS who achieve disease control following induction therapy with PBC. In the PARAMOUNT study (14), a significant reduction in the risk of disease progression was observed in the pemetrexed group compared to the placebo group $(\mathrm{HR}=0.62$; 95\% CI: 0.49-0.79; P<0.0001). The median PFS was 4.1 months (95\% CI:3.2-4.6 months) for pemetrexed and 2.8 months (95\% CI: 2.6-3.1 months) for placebo. The median OS was 13.9 months for pemetrexed and 11.0 months for placebo. Pemetrexed maintenance therapy resulted in a $22 \%$ reduction in the risk of death $(\mathrm{HR}=0.78,95 \% \mathrm{CI}: 0.64-0.96 ; \mathrm{P}=0.0195)$. In the AVAPERL study (15), after a median follow-up of 8.1 months, the PFS from random assignment was significantly improved in the bevacizumab plus pemetrexed group compared to that in the bevacizumab alone group (median PFS: 3.7 vs. 7.4 months, respectively; $\mathrm{HR}=0.48 ; 95 \% \mathrm{CI}: 0.35-0.66 ; \mathrm{P}<0.001$ ).
However, not all patients require maintenance therapy, since some patients experience a long PFS after initial PBC without any sign of disease progression. For those who do not receive maintenance therapy after induction therapy, a PBC rechallenge may be the optimal second-line option, provided the PFS1 or TFS is sufficiently long, as demonstrated by the present study. These two modalities, maintenance and rechallenge therapy, may apply to different patients. We hypothesized that, for patients who experience disease progression within a short time after PBC induction, maintenance therapy is required, whereas patients who achieve long-term SD are candidates for rechallenge therapy. However, this hypothesis requires confirmation by further studies.

There were certain limitations to our study. First, all the enrolled patients responded well to initial PBC, resulting in a longer PFS1 (median, 10.2 months) compared to that previously reported by studies on Asian patients (4.3-6.8 months) $(16,17)$. In addition, due to the relatively small sample size, we did not observe any significant differences between PBC-sensitive and PBC-resistant groups in the analyses of molecular markers.

To the best of our knowledge, this study was the first on PBC rechallenge in lung cancer. We demonstrated that rechallenging with $\mathrm{PBC}$ is tolerable and may be a viable option for patients with advanced non-squamous NSCLC with a good PS and a PFS1 of $\geq 10$ months or a TFS of $\geq 3$ months after initial treatment with $\mathrm{PBC}$. Our findings may provide another effective strategy for patients who had benefited from PBC induction chemotherapy but did not receive pemetrexed maintenance therapy. Further large-scale clinical trials are required to determine the best criteria for $\mathrm{PBC}$ rechallenge and identify potential biomarkers that predict sensitivity to pemetrexed.

\section{Acknowledgements}

This study was supported by the National Natural Science Foundation Fund for Distinguished Young Scholars (81025012); the National Natural Science Foundation Key Program (81330062); and the Beijing Health Systems Academic Leader (2011-2-22). We would like to thank our colleagues at the Department of Thoracic Medical Oncology, Peking University School of Oncology, Beijing Cancer Hospital and Institute, for their support.

\section{References}

1. Scagliotti GV, Parikh P, von Pawel J, et al: Phase III study comparing cisplatin plus gemcitabine with cisplatin plus pemetrexed in chemotherapy-naive patients with advanced-stage non-small-cell lung cancer. J Clin Oncol 26: 3543-3551, 2008.

2. Hanna N, Shepherd FA, Fossella FV, et al: Randomized phase III trial of pemetrexed versus docetaxel in patients with non-small-cell lung cancer previously treated with chemotherapy. J Clin Oncol 22: 1589-1597, 2004.

3. Vogelzang NJ, Rusthoven JJ, Symanowski J, et al: Phase III study of pemetrexed in combination with cisplatin versus cisplatin alone in patients with malignant pleural mesothelioma. J Clin Oncol 21: 2636-2644, 2003.

4. Ceresoli GL, Zucali PA, De Vincenzo F, et al: Retreatment with pemetrexed-based chemotherapy in patients with malignant pleural mesothelioma. Lung Cancer 72: 73-77, 2011.

5. Zucali PA, Simonelli M, Michetti G, et al: Second-line chemotherapy in malignant pleural mesothelioma: results of a retrospective multicenter survey. Lung Cancer 75: 360-367, 2012. 
6. Bearz A, Talamini R, Rossoni G, et al: Re-challenge with pemetrexed in advanced mesothelioma: a multi-institutional experience. BMC Res Notes 5: 482, 2012.

7. Eisenhauer EA, Therasse P, Bogaerts J, et al: New response evaluation criteria in solid tumours: revised RECIST guideline (version 1.1). Eur J Cancer 45: 228-247, 2009.

8. Bai H, Wang Z, Chen K, et al: Influence of chemotherapy on EGFR mutation status among patients with non-small-cell lung cancer. J Clin Oncol 30: 3077-3083, 2012.

9. Chen CY, Chang YL, Shih JY, et al: Thymidylate synthase and dihydrofolate reductase expression in non-small cell lung carcinoma: the association with treatment efficacy of pemetrexed. Lung Cancer 74: 132-138, 2011.

10. Christoph DC, Asuncion BR, Mascaux C, et al: Folylpolyglutamate synthetase expression is associated with tumor response and outcome from pemetrexed-based chemotherapy in malignant pleural mesothelioma. J Thorac Oncol 7: 1440-1448, 2012.

11. Monica V, Scagliotti GV, Ceppi P, et al: Differential thymidylate synthase expression in different variants of large-cell carcinoma of the lung. Clin Cancer Res 15: 7547-7552, 2009.

12. Pfisterer J and Ledermann JA: Management of platinum-sensitive recurrent ovarian cancer. Semin Oncol 33 (Suppl 6): S12-S16, 2006 .
13. Garassino MC, Torri V, Michetti G, et al: Outcomes of small-cell lung cancer patients treated with second-line chemotherapy: A multi-institutional retrospective analysis. Lung Cancer 72: 378-383, 2011.

14. Paz-Ares LG, de Marinis F, Dediu M, et al: PARAMOUNT: Final overall survival results of the phase III study of maintenance pemetrexed versus placebo immediately after induction treatment with pemetrexed plus cisplatin for advanced nonsquamous non-small-cell lung cancer. J Clin Oncol 31: 2895-2902, 2013.

15. Barlesi F, Scherpereel A, Rittmeyer A, et al: Randomized phase III trial of maintenance bevacizumab with or without pemetrexed after first-line induction with bevacizumab, cisplatin, and pemetrexed in advanced nonsquamous non-small-cell lung cancer: AVAPERL (MO22089). J Clin Oncol 31: 3004-3011, 2013.

16. Kawano Y, Ohyanagi F, Yanagitani N, et al: Pemetrexed and cisplatin for advanced non-squamous non-small cell lung cancer in Japanese patients: phase II study. Anticancer Res 33: 3327-3333, 2013.

17. Xu B, Liu P, Yin Y, Liu P and Shu Y: Pemetrexed as the firstline therapy for Chinese patients with advanced non-squamous non-small-cell lung cancer. Biomed Pharmacother 67: 763-769, 2013. 